\title{
Bacteremic community-acquired pneumonia due to Klebsiella pneumoniae: Clinical and microbiological characteristics in Taiwan, 2001-2008
}

\author{
Yi-Tsung Lin ${ }^{1,2 \dagger}$, Yuan-Yu Jeng ${ }^{1 \dagger}$, Te-Li Chen ${ }^{1,2}$, Chang-Phone Fung ${ }^{1,2^{*}}$
}

\begin{abstract}
Background: Klebsiella pneumoniae is the major cause of community-acquired pyogenic infections in Taiwan. This retrospective study evaluated the clinical and microbiological characteristics of bacteremic community-acquired pneumonia due to K. pneumoniae in Taiwanese adults.

Methods: The clinical characteristics of bacteremic community-acquired pneumonia (CAP) in adults due to K. pneumoniae were compared to those of adults with bacteremic CAP due to Streptococcus pneumoniae at a tertiary medical center in Taiwan from 2001-2008. Risk factors for mortality of bacteremic CAP due to K. pneumoniae were analyzed. All clinical isolates of $K$. pneumoniae were examined for capsular serotypes, hypermucoviscosity phenotype, aerobactin and rmpA gene.
\end{abstract}

Results: K. pneumoniae was the dominant cause of bacteremic CAP and was associated with a more fulminant course and a worse prognosis than bacteremic CAP due to Streptococcus pneumoniae. Initial presentation with septic shock and respiratory failure were independent risk factors for both early and total mortality. Serotype K1 and K2 comprised around half of all isolates. There were no significant differences in the clinical characteristics of patients with bacteremic CAP due to K1/K2 and non-K1/K2 isolates. Hypermucoviscosity phenotype as well as the aerobactin and rmpA genes were highly prevalent in the $K$. pneumoniae isolates.

Conclusions: $K$. pneumoniae continued to be the dominant cause of bacteremic CAP in Taiwanese adults during 2001-2008. Initial presentation with septic shock and respiratory failure were independent risk factors for both early and total mortality from K. pneumoniae bacteremic CAP. Serotypes K1/K2 comprised around half of all isolates, but did not predispose patients to a poor clinical outcome. Physicians should be aware of the poor prognosis of any patient with bacteremic K. pneumoniae CAP and monitor these patients more closely.

\section{Background}

Klebsiella pneumoniae is the major cause of liver abscess, Gram-negative bacillary meningitis, brain abscess, lung abscess, thoracic empyema, prostatic abscess, deep neck infection and complicated skin and soft tissue infections in Taiwan [1], and comprised 4.8\% of the common causative pathogens of communityacquired pneumonia (CAP) in a multi-center survey [2]. Although K. pneumoniae is generally considered an infrequent cause of CAP in the United States and

\footnotetext{
* Correspondence: cpfung@vghtpe.gov.tw

† Contributed equally

'Division of Infectious Diseases, Department of Medicine, Taipei Veterans General Hospital, Taipei, Taiwan

Full list of author information is available at the end of the article
}

Europe, a large prospective study showed that $K$. pneumoniae was not only a frequent etiology of severe CAP, but also an independent risk factor for mortality [3].

A number of studies have suggested that bacteremia is a risk factor for death in patients with CAP [4-7]. Although previous studies investigated the characteristics and treatment strategy for bacteremic pneumococcoal pneumonia, few studies of bacteremic CAP due to $K$. pneumoniae have been reported and most of these were conducted before 2000 [8-10]. In one global study, pneumonia accounted for $29 \%$ and $62 \%$ of all cases of community-acquired K. pneumoniae bacteremia in Taiwan and South Africa, respectively; however, only four cases of community-acquired bacteremic K. pneumoniae 
pneumonia were seen in the United States, Argentina, Europe, or Australia [10].

The present study compared the clinical characteristics of bacteremic CAP due to K. pneumoniae with the characteristics of bacteremic CAP due to Streptococcus pneumoniae and the virulence factors of invasive $K$ pneumoniae isolates in a tertiary medical center over an 8 -year period in Taiwan.

\section{Methods}

\section{Patient selection}

Microbiology records were used to identify consecutive patients with bacteremic CAP admitted between January 2001 and December 2008 at Taipei Veterans General Hospital, a 2,900-bed tertiary medical center in northern Taiwan. The medical records of patients with bacteremic CAP due to either K. pneumoniae or S. pneumoniae were reviewed. CAP was defined as an acute lower respiratory tract infection characterized by the following: (1) an acute pulmonary infiltrate evident on CXR and compatible with pneumonia, (2) confirmatory findings of the clinical examination, and (3) acquisition of the infection outside a hospital, long-term care facility, or nursing home [11]. Bacteremic CAP was defined as the presence of at least one blood culture sampled within $48 \mathrm{~h}$ of presentation in the hospital, yielding a pathogen presumed to be the cause of the pneumonia [11], and the absence of other infectious sources which could account for the bacteremia. Patients younger than 18 years old were excluded. The diagnosis was reconfirmed by 2 infectious diseases specialists. The institutional review board of the Taipei Veterans General Hospital approved this study.

\section{Data collection and definition}

The following data were collected for each patient: demographic characteristics, underlying diseases or risk factors, clinical variables present at admission, Acute Physiologic and Chronic Health Evaluation (APACHE) II score, pneumonia severity index (PSI) score [12], laboratory data, empirical antibiotics received, chest radiograph reports, days of hospitalization, deaths less than 48 hrs after admission, and in-hospital death. Concordant antibiotic therapy was defined as initial antibiotic therapy including any antibiotic to which the infecting organism was sensitive based on the microbiology report. The diagnosis of septic shock was based on a systolic blood pressure of $<90 \mathrm{mmHg}$ and peripheral hypoperfusion with clinical or bacteriological evidence of uncontrolled infection. Neurologic diseases encompassed stroke and head injury. Chronic obstructive pulmonary diseases, bronchiectasis, and old pulmonary tuberculosis belonged to the subgroup of chronic lung diseases. The outcome of bacteremic CAP was defined as in-hospital mortality measured at 28 days after onset of bacteremic CAP. Early mortality was defined as death less than $48 \mathrm{hrs}$ after onset of bacteremic CAP.

\section{Microbiology laboratory procedures}

The VITEK 2 system (bioMérieux, Marcy l'Etoile, France) was used to confirm bacterial identifications. The antimicrobial susceptibility of $K$. pneumoniae was tested by the disk diffusion method and interpretations were made according to the guidelines of the Clinical and Laboratory Standards Institute [13].

\section{Serotyping and detection of hypermucoviscosity phenotype, aerobactin, and rmpA gene}

All isolates of $K$. pneumoniae causing bacteremic CAP were serotyped by a countercurrent immunoelectrophoresis method as described previously [14]. Antisera were provided by the Gram Negative Serotyping Unit of the Laboratory of Health Care Associated Infection (Health Protection Agency Centre for Infections, London). K. pneumoniae ATCC9997 (K2) was used as a control strain. All isolates with K1 and K2 serotypes were confirmed by PCR for serotype-specific targets within the $\mathrm{K} 1$ and $\mathrm{K} 2$ cps clusters as described previously [15]. Using crude genomic DNA as the template, we performed PCRs to amplify the rmpA and aerobactin genes as described previously [16]. Strains with the hypermucoviscosity phenotype were defined by a positive string test as described previously [16].

\section{Statistics}

Contingency data were analyzed by 2-tailed chi-square test or Fisher's exact test, and continuous data were analyzed by Student's $\mathrm{t}$ test or the Mann-Whitney U test. A $p$ value of $<0.05$ was considered to be statistically significant, and all probabilities were 2-tailed. Variables with $p$ values of $<0.10$ in univariate analyses subsequently entered in the multivariate analysis. The multivariate logistic regression model was used to evaluate the risk factors for mortality in bacteremic CAP caused by $K$. pneumoniae. All statistical analyses were performed with SPSS, version 15.0 for Windows (SPSS).

\section{Results}

\section{Clinical characteristics of patients with bacteremic CAP} due to K. pneumoniae

During the study period, 95 patients with bacteremic CAP due to either K. pneumoniae or S. pneumoniae were identified from 148 consecutive patients with bacteremic CAP. Excluding two patients whose infections were determined to be caused by polymicrobial pathogens mixed with S. pneumoniae, the remaining 93 
bacteremic pneumonia patients were divided into two groups with $K$. pneumoniae or S. pneumoniae as the causative pathogen. During the study period, bacteremic CAP was more frequently caused by $K$. pneumoniae than S. pneumoniae. The clinical characteristics of the patients with infections caused by these two pathogens are compared in Table 1 . Patients with $K$. pneumoniae infection had a significantly higher mortality rate $(55.1 \%$ vs. $27.3 \%$, respectively, $P=0.007$ ), a significantly higher early mortality rate $(36.7 \%$ vs. $13.6 \%$, respectively, $P=$ 0.011 ), a significantly higher prevalence of PSI Risk Class V $(89.8 \%$ vs. $61.4 \%$, respectively, $P=0.001)$, a significantly higher prevalence of ICU admission $(57.1 \%$ vs. $31.8 \%$, respectively, $P=0.014)$, a significantly higher prevalence of initial presentation with respiratory failure (61.2\% vs. $31.8 \%$, respectively, $P=0.005)$, a significantly higher prevalence of APACHE II score $>25$ (71.4\% vs. $29.5 \%$, respectively, $P<0.001)$ and a greater likelihood of initial presentation with septic shock (51\% vs. $31.8 \%$, respectively, $P=0.061$ ). None of the 49 cases of bacteremic CAP due to $K$. pneumoniae had concurrent liver abscess or other metastatic infection. All patients with bacteremic K. pneumoniae CAP received concordant antibiotic treatment according to the results of susceptibility testing.
Laboratory findings for bacteremic community-acquired pneumonia due to $K$. pneumoniae

Table 2 compares the chest radiography and laboratory findings between patients with bacteremic K. pneumoniae and S. pneumoniae CAP. Patients with bacteremic $K$. pneumoniae CAP had a higher prevalence of bilateral involvement on chest radiography $(65.3 \%$ vs. $22.7 \%$, respectively, $p<0.001$ ) and a lower leukocyte count $(11.6 \pm 6.7 \times 103 / \mu \mathrm{L}$ vs. $14.4 \pm 7.4 \times 103 / \mu \mathrm{L}$, respectively, $P=0.044)$.

\section{Factors associated with mortality in K. pneumoniae bacteremic CAP}

The results of univariate analyses of variables related to death in bacteremic CAP due to K. pneumoniae are listed in Table 3. In the logistic regression analysis, initial presentation with shock (OR 16.9, 95\% CI 3.0-94.4, $P=$ 0.001 ) and respiratory failure (OR 13.3, 95\% CI 2.3-77.6, $P=0.004)$ were the only independent factors associated with fatal outcome. Early mortality compromised two thirds of deaths in patients with bacteremic CAP due to K. pneumoniae. Univariate analyses of variables related to early mortality and subsequent logistic regression analysis revealed that initial presentation with septic shock (OR 18.9, 95\% CI 3.0-118.5, $p=0.002$ ) and respiratory failure

Table 1 Comparison of clinical characteristics of patients with bacteremic $K$. pneumoniae and S. pneumoniae community-acquired pneumonia

\begin{tabular}{|c|c|c|c|}
\hline Characteristic & $\begin{array}{c}\text { K. pneumoniae } \\
(n=49) \\
\text { No. }(\%)\end{array}$ & $\begin{array}{c}\text { S. pneumoniae } \\
(n=44) \\
\text { No. }(\%)\end{array}$ & $p$-value \\
\hline Age, mean years $\pm S E$ & $73 \pm 14$ & $76 \pm 11$ & 0.893 \\
\hline Male sex & 39 (79.6) & 39 (88.6) & 0.236 \\
\hline \multicolumn{4}{|l|}{ Predisposing condition or risk factor } \\
\hline Diabetes mellitus & $17(34.7)$ & $11(25)$ & 0.309 \\
\hline Malignancy & $14(28.6)$ & $11(25)$ & 0.698 \\
\hline Neurologic disorders & $12(24.5)$ & $12(27.3)$ & 0.759 \\
\hline Chronic kidney disease stage $\geqq 4$ & $13(26.5)$ & $13(29.5)$ & 0.746 \\
\hline Chronic lung diseases & $23(46.9)$ & $21(47.7)$ & 0.939 \\
\hline Liver cirrhosis & $4(8.2)$ & $3(6.8)$ & 1.000 \\
\hline Alcoholism & $5(10.2)$ & $1(2.3)$ & 0.207 \\
\hline Autoimmune disease & $3(6.1)$ & $1(2.3)$ & 0.619 \\
\hline No underlying diseases & $3(6.1)$ & $5(11.4)$ & 0.470 \\
\hline APACHE II score $>25$ & $35(71.4)$ & $13(29.5)$ & $<0.001$ \\
\hline Days of hospitalization & $28 \pm 24$ & $21 \pm 15$ & 0.929 \\
\hline Initial presentation with septic shock & $25(51)$ & $14(31.8)$ & 0.061 \\
\hline Initial presentation with respiratory failure & $30(61.2)$ & $14(31.8)$ & 0.005 \\
\hline PSI Risk Class V & $44(89.8)$ & $27(61.4)$ & 0.001 \\
\hline ICU admission & $28(57.1)$ & $14(31.8)$ & 0.014 \\
\hline Concurrent lung abscess & $3(6.1)$ & $0(0)$ & 0.244 \\
\hline Early mortality (deaths $\leqq 48$ hrs) & $18(36.7)$ & $6(13.6)$ & 0.011 \\
\hline In-hospital mortality & $27(55.1)$ & $12(27.3)$ & 0.007 \\
\hline
\end{tabular}

Values given as mean \pm SD or No. of patients (\%).APACHE, Acute Physiologic and Chronic Health Evaluation; PSI, Pneumonia Severity Index; ICU, intensive care unit. 
Table 2 Comparison of chest radiography and laboratory findings of patients with bacteremic K. pneumoniae and S. pneumoniae community-acquired pneumonia

\begin{tabular}{|c|c|c|c|}
\hline Characteristic & $\begin{array}{c}\text { K. pneumoniae } \\
(\mathrm{n}=49) \\
\text { No. }(\%)\end{array}$ & $\begin{array}{l}\text { S. pneumoniae } \\
(\mathrm{n}=44) \\
\text { No. }(\%)\end{array}$ & $p$-value \\
\hline \multicolumn{4}{|l|}{ Chest radiography at admission } \\
\hline Unilateral involvement & $17(34.7)$ & $34(77.3)$ & $<0.001$ \\
\hline Bilateral involvement & $32(65.3)$ & $10(22.7)$ & $<0.001$ \\
\hline Cavity lesion & $3(6.1)$ & $1(2.3)$ & 0.619 \\
\hline \multicolumn{4}{|l|}{ Initial laboratory value } \\
\hline Leucocyte count, $\times 103 / \mu \mathrm{L}$ & $11.6 \pm 6.7$ & $14.4 \pm 7.4$ & 0.044 \\
\hline Platelet, $\times 103 / \mu \mathrm{L}$ & $212 \pm 101$ & $196 \pm 107$ & 0.969 \\
\hline Albumin, $\mathrm{g} / \mathrm{dL}$ & $2.6 \pm 0.6$ & $2.8 \pm 0.6$ & 0.072 \\
\hline C-reactive protein, $\mathrm{mg} / \mathrm{dL}$ & $21.8 \pm 11.2$ & $24.9 \pm 1.5$ & 0.664 \\
\hline Serum creatinine, $\mathrm{mg} / \mathrm{dL}$ & $2.3 \pm 1.9$ & $2.3 \pm 1.4$ & 0.186 \\
\hline Total biliburin, mg/dL & $1.2 \pm 2.7$ & $1.1 \pm 1.3$ & 0.809 \\
\hline Glucose, mg/dL & $220 \pm 168$ & $208 \pm 202$ & 0.528 \\
\hline
\end{tabular}

Values given as mean \pm SD or No. of patients (\%).

(OR 22.6, 95\% CI 2.2-229.9, $P=0.008$ ) were as well the only independent factors associated with early mortality.

\section{Susceptibility testing}

All $49 \mathrm{~K}$. pneumoniae isolates showed uniform resistance to ampicillin and susceptibility to a number classes of antibiotics including cephalosporins, aminoglycosides, and fluoroquinolones.
Capsular serotypes and virulence factors of $K$. pneumoniae Serotypes K1 and K2 accounted for 14.3\% (7/49) and $38.8 \%(19 / 49)$ of all $K$. pneumoniae isolates, respectively. The prevalence of non $\mathrm{K} 1 / \mathrm{K} 2$ serotypes of 23 isolates revealed $\mathrm{K} 5(\mathrm{n}=3), \mathrm{K} 20(\mathrm{n}=2)$, and unidentified type $(\mathrm{n}=18)$. There were no significant difference in the clinical characteristics of patients with bacteremic CAP due to $\mathrm{K} 1 / \mathrm{K} 2$ and non-K1/K2 isolates (Table 4). All K1/

Table 3 Risk factors for mortality in bacteremic CAP caused by K. pneumoniae

\begin{tabular}{|c|c|c|c|}
\hline Characteristic & $\begin{array}{c}\text { Survivors } \\
(\mathrm{n}=22) \\
\text { No. }(\%)\end{array}$ & $\begin{array}{c}\text { Non-survivors } \\
(\mathrm{n}=27) \\
\text { No. }(\%)\end{array}$ & $p$-value \\
\hline Age $>65$ years & $15(68.2)$ & $22(81.5)$ & 0.282 \\
\hline Male & $19(86.4)$ & $20(74.1)$ & 0.288 \\
\hline Initial presentation with septic shock & $4(18.2)$ & $21(77.8)$ & $<0.001$ \\
\hline Initial presentation with respiratory failure & $7(31.8)$ & $23(85.2)$ & $<0.001$ \\
\hline APACHE II score $>25$ & $10(45.5)$ & $25(92.6)$ & $<0.001$ \\
\hline PSI Risk Class V & $18(81.8)$ & $26(96.3)$ & 0.160 \\
\hline ICU admission & $10(45.5)$ & $18(66.7)$ & 0.136 \\
\hline Malignancy & $5(22.7)$ & $9(33.3)$ & 0.414 \\
\hline Diabetes mellitus & $10(45.5)$ & $7(25.9)$ & 0.153 \\
\hline Liver cirrhosis & $1(4.5)$ & $3(11.1)$ & 0.617 \\
\hline Chronic kidney disease stage $\geqq 4$ & $6(27.3)$ & $7(25.9)$ & 0.915 \\
\hline Chronic lung diseases & $12(54.5)$ & $11(40.7)$ & 0.336 \\
\hline Neurologic disorders & $7(31.8)$ & $5(18.5)$ & 0.282 \\
\hline Autoimmune disease & $1(4.5)$ & $1(3.8)$ & 1.000 \\
\hline Alcoholism & $2(9.1)$ & $3(11.1)$ & 1.000 \\
\hline No underlying diseases & $1(4.0)$ & $2(7.4)$ & 0.609 \\
\hline Chest radiography_-bilateral involment & $14(63.6)$ & $18(66.7)$ & 0.825 \\
\hline Leucopenia $^{a}$ & $2(9.1)$ & $4(14.8)$ & 0.678 \\
\hline Thrombocytopenia & $2(9.1)$ & $9(33.3)$ & 0.043 \\
\hline C-reactive protein, $\mathrm{mg} / \mathrm{dL}$ & $20.9 \pm 12.1$ & $22.7 \pm 10.6$ & 0.482 \\
\hline
\end{tabular}

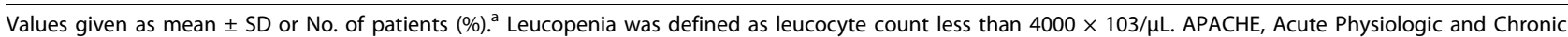
Health Evaluation; PSI, Pneumonia Severity Index; ICU, intensive care unit. 
Table 4 Relation of $K$. pneumoniae serotype to clinical characteristics

\begin{tabular}{|c|c|c|c|c|}
\hline Characteristic & $\begin{array}{c}\text { Serotype K1, } \\
n=7 \\
\text { No. (\%) }\end{array}$ & $\begin{array}{c}\text { Serotype K2, } \\
n=19 \\
\text { No. }(\%)\end{array}$ & $\begin{array}{c}\text { Serotype non-K1/K2, } \\
n=23 \\
\text { No. (\%) }\end{array}$ & $p$-value ${ }^{a}$ \\
\hline Age $>65$ years & $5(71.4)$ & $14(73.7)$ & $18(78.3)$ & 0.674 \\
\hline Male sex & $4(57.1)$ & $15(78.9)$ & $20(87)$ & 0.229 \\
\hline \multicolumn{5}{|l|}{ Underlying disease or risk factor } \\
\hline Malignancy & $1(14.3)$ & $5(26.3)$ & $8(34.8)$ & 0.365 \\
\hline Diabetes mellitus & $5(71.4)$ & $6(31.6)$ & $6(26.1)$ & 0.234 \\
\hline Liver cirrhosis & $0(0)$ & $2(10.5)$ & $2(8.7)$ & 1.000 \\
\hline Chronic kidney disease stage $\geqq 4$ & $1(14.3)$ & $5(26.3)$ & $7(30.4)$ & 0.560 \\
\hline Chronic lung diseases & $2(28.6)$ & $10(52.6)$ & $11(47.8)$ & 0.907 \\
\hline Neurologic disorders & $3(42.9)$ & $2(10.5)$ & $7(30.4)$ & 0.363 \\
\hline Autoimmune disease & $0(0)$ & $2(10.5)$ & $1(4.3)$ & 1.000 \\
\hline Alcoholism & $1(14.3)$ & $2(10.5)$ & $2(8.7)$ & 1.000 \\
\hline CXR-bilateral involvement & $6(85.7)$ & $12(63.2)$ & $14(60.9)$ & 0.539 \\
\hline Leucopenia b & $1(14.3)$ & $3(15.8)$ & $2(8.7)$ & 0.671 \\
\hline Concurrent lung abscess & $0(0)$ & $2(10.5)$ & $1(4.3)$ & 1.000 \\
\hline Initial presentation with septic shock & $4(57.1)$ & $10(52.6)$ & $11(47.8)$ & 0.674 \\
\hline Initial presentation with respiratory failure & $4(57.1)$ & $12(63.2)$ & $14(60.9)$ & 0.962 \\
\hline APACHE-II score $>25$ & $5(71.4)$ & $15(78.9)$ & $15(65.2)$ & 0.365 \\
\hline PSI Risk Class V & $7(100)$ & $17(89.5)$ & $20(87)$ & 0.655 \\
\hline ICU admission & $3(42.9)$ & $12(63.2)$ & $13(56.5)$ & 0.934 \\
\hline Early deaths $\leqq 48$ hrs & $3(42.9)$ & $7(36.8)$ & $8(34.8)$ & 0.790 \\
\hline In-hospital death & $4(57.1)$ & $11(57.9)$ & $12(52.2)$ & 0.698 \\
\hline
\end{tabular}

Values given as No. of patients (\%). ${ }^{a}$ Serotype K1/K2 vs. Serotype non-K1/K2.

${ }^{b}$ Leucopenia was defined as leucocyte count less than $4000 \times 103 / \mu \mathrm{L}$. APACHE, Acute Physiologic and Chronic Health Evaluation; PSI, Pneumonia Severity Index, ICU: intensive care unit.

K2 isolates $(\mathrm{n}=26)$ and 20 out of 23 non-K1/K2 isolates were positive for the hypermucoviscosity phenotype, aerobactin and rmpA genes. Three other non-K1/ $\mathrm{K} 2$ isolates were neither hypermucoviscous nor positive for aerobactin or $\operatorname{rmp} A$ genes. There were no statistically significant differences in the prevalence of the hypermucoviscosity phenotype, the aerobactin and rmpA genes between the $\mathrm{K} 1 / \mathrm{K} 2$ and non-K1/K2 isolates $P=0.096)$.

\section{Discussion}

K. pneumoniae is the major cause of pyogenic infections worldwide, especially in Taiwan [1]. The present study further highlights the role of $K$. pneumoniae as a dominant pathogen in bacteremic CAP in Taiwan. A previous investigation of bacteremic CAP in Taiwan from June 1988 to September 1991 found that K. pneumoniae was the most frequently isolated sole pathogen (34.1\%) [9]. The present study demonstrated that $K$. pneumoniae was the dominant pathogen from cases of bacteremic CAP (33.1\%) in a large tertiary-care hospital in Taiwan between 2001 and 2008. Though conducted in a single institute, the findings of the present study are compatible with the previous cohort [9], and again reflect the highly endemic nature of K. pneumoniae infections in Taiwan [10,17].
Previous studies in Taiwan found that approximately $40-50 \%$ of community-acquired $K$. pneumoniae bacteremia was associated with diabetes mellitus $[10,18]$, and patients with liver cirrhosis were more likely to develop K. pneumoniae bacteremia [19]. In addition, bacteremic CAP due to K. pneumoniae was significantly associated with alcoholism [10]. However, in the present study, the most common underlying diseases were chronic lung diseases, which may be explained by the older age of the study population. Diabetes, a classic risk factor for community-acquired $K$. pneumoniae infection, did not predispose patients to bacteremic K. pneumoniae CAP in this study. This finding suggests that the mechanism responsible for the susceptibility to $K$. pneumoniae infection may be different for bacteremic CAP than for other clinical manifestations, such as liver abscess. Further study is needed to define the risk factors which predispose patients to the development of bacteremic K. pneumoniae CAP.

There has been limited study of clinical characteristics of bacteremic $K$. pneumoniae CAP in the last decade. In this study, we found that the severity of CAP and the prevalence of bilateral involvement on chest X-ray were significantly greater in patients with bacteremic K. pneumoniae CAP. Patients with bacteremic K. pneumoniae 
CAP also had worse clinical course than patients infected with S. pneumoniae. Knowledge of the different characteristics of $K$. pneumoniae and S. pneumoniae bacteremic CAP is of great importance for physicians in endemic areas such as Taiwan. It is also vital for the medical community in which physicians are constantly faced with the diagnostic and prognostic challenges of CAP patients with uncommon pathogens. A previous study found patients infected with $K$. pneumoniae had increased frequency of lower platelet count, leucopenia, higher serum albumin, and male sex [8]. The lower leucocyte count of patients with $K$. pneumoniae bacteremic CAP in the present study is consistent with these findings.

A study of ICU cases with bacteremic CAP in South Africa from January 1982 through July 1985 found that mortality rate was $80 \%$ in patients with $K$. pneumoniae infections and $67 \%$ in patients with S. pneumoniae infections [8]. Another study from 1996 through 1997 found that death rates from bacteremic CAP due to $K$. pneumoniae were $54 \%$ in Taiwan and 56\% in South Africa [10]. Mortality rate from bacteremic CAP due to K. pneumoniae remained high $(55 \%)$ in this study despite advances in antimicrobial agents and supportive care. The independent risk factors for mortality from bacteremic CAP due to $K$. pneumoniae in this study were initial presentation with septic shock and respiratory failure. Previous study found that the first 48 hours of evolution of CAP were critical [20] and early mortality was also high in the present study. We also found that initial presentation with septic shock and respiratory failure were the only factors associated with early mortality. A previous study found that alcoholism was a risk factor for poor outcome of bacteremic CAP due to K. pneumoniae [21]. However, the present study did not find any association between specific underlying disease subgroups and mortality due to K. pneumoniae bacteremic CAP. Physicians should be aware of the high mortality rate in patients with bacteremic K. pneumoniae CAP and monitor patients more closely.

In this study, capsular serotype $\mathrm{K} 1$ and $\mathrm{K} 2$ comprised $14.3 \%$ and $38.8 \%$, respectively, of all $K$. pneumoniae isolates. Previous studies reported that serotypes K1 and K2 were more common in isolates from community-acquired infections in Taiwan, South Africa, and Singapore than from hospital-acquired isolates in these countries or elsewhere $[17,22]$. Metastatic infection is an important complication of $K$. pneumoniae liver abscess in Taiwan, and certain capsular serotypes of $K$. pneumoniae including K1 and $\mathrm{K} 2$ are thought to play an important role in the development of distal metastatic infections, generally being considered the predominant virulent strains [22-24]. Our previous study indicated that serotype $\mathrm{K} 1$ and $\mathrm{K} 2 \mathrm{com}-$ prised $59 \%$ of K. pneumonaie in community-acquired thoracic empyema or complicated parapneumonic effusion but did not predispose patients to poor outcome compared with other non-K1/K2 serotypes [25]. In the present study, patients with $\mathrm{K} 1 / \mathrm{K} 2$ serotype $K$. pneumoniae isolates also did not have a worse outcome than those with non-K1/K2 serotype of $K$. pneumoniae isolates, and none of the patients with bacteremic CAP due to K. pneumoniae developed metastatic infection. This finding suggests that pulmonary infection with $K$. pneumoniae might carry little risk for distant metastasis even in patients with bloodstream infection. It also suggests that though serotypes $\mathrm{K} 1$ and $\mathrm{K} 2$ were the prevalent strains in communityacquired pulmonary infection due to K. pneumonia, serotypes were not related to the clinical characteristics and prognosis. The current study found the high prevalence of hypermucoviscosity phenotype as well as the aerobactin and $r m p A$ genes in K. pneumoniae isolates, which was consistent to the previous investigation of liver abscess strains [16]. The high frequency of coexistence of the aerobactin and $r m p A$ genes in the strains causing bacteremic CAP was in agreement with previous reports of liver abscess strains and strains causing bloodstream infections $[16,17]$.

\section{Conclusions}

In conclusion, during 2001-2008, K. pneumoniae continued to be the dominant cause of bacteremic CAP in Taiwanese adults in this tertiary-care hospital. The K. pneumoniae bacteremic CAP had a higher mortality than S. pneumoniae bacteremic CAP. Initial presentation with septic shock and respiratory failure were independent risk factors for both early and total mortality from $K$. pneumoniae bacteremic CAP. Serotypes K1/K2 comprised around half of all isolates, but did not predispose patients to a poor clinical outcome. Physicians should be aware of the poor prognosis of any patient with bacteremic $K$. pneumoniae CAP and monitor these patients closely.

\section{Abbreviations \\ CAP: community-acquired pneumonia; 95\% Cl: 95\% confidence interval; OR: odds ratio; APACHE: Acute Physiologic and Chronic Health Evaluation; PSI: pneumonia severity index; ICU: intensive care unit.}

\section{Acknowledgements}

Financial support: This study was supported by grants from Taipei Veterans General Hospital.

\section{Author details}

${ }^{1}$ Division of Infectious Diseases, Department of Medicine, Taipei Veterans General Hospital, Taipei, Taiwan. ${ }^{2}$ Institute of Clinical Medicine, School of Medicine, National Yang-Ming University, Taipei, Taiwan.

\section{Authors' contributions}

LYT, JYY, and FCP conceived of the study, and participated in its design and coordination. LYT, JYY, and FCP performed the experiments. LYT, JYY, CTL and FCP reviewed the medical records. LYT, JYY, CTL and FCP analyzed and interpreted the data. LYT, JYY, and FCP drafted the manuscript. All authors read and approved the final manuscript. 


\section{Competing interests}

The authors declare that they have no competing interests.

Received: 13 June 2010 Accepted: 25 October 2010

Published: 25 October 2010

\section{References}

1. Chang CM, Lee HC, Lee NY, Lee IW, Wu CJ, Chen PL, Lee CC, Ko NY, Ko WC: Community-acquired Klebsiella pneumoniae complicated skin and soft-tissue infections of extremities: emphasis on cirrhotic patients and gas formation. Infection 2008, 36:328-334.

2. Lauderdale TL, Chang FY, Ben RJ, Yin HC, Ni YH, Tsai JW, Cheng SH, Wang JT, Liu YC, Cheng YW, et al: Etiology of community acquired pneumonia among adult patients requiring hospitalization in Taiwan. Respiratory Medicine 2005, 99:1079-1086.

3. Paganin F, Lilienthal F, Bourdin A, Lugagne N, Tixier F, Genin R, Yvin JL: Severe community-acquired pneumonia: assessment of microbial aetiology as mortality factor. Eur Respir J 2004, 24:779-785.

4. Waterer GW, Somes GW, Wunderink RG: Monotherapy may be suboptimal for severe bacteremic pneumococcal pneumonia. Arch Intern Med 2001, 161:1837-1842.

5. Plouffe JF, Breiman RF, Facklam RR: Bacteremia with Streptococcus pneumoniae. Implications for therapy and prevention. Franklin County Pneumonia Study Group. JAMA 1996, 275:194-198.

6. Lujan M, Gallego M, Fontanals D, Mariscal D, Rello J: Prospective observational study of bacteremic pneumococcal pneumonia: Effect of discordant therapy on mortality. Crit Care Med 2004, 32:625-631.

7. Chokshi R, Restrepo MI, Weeratunge N, Frei CR, Anzueto A, Mortensen EM: Monotherapy versus combination antibiotic therapy for patients with bacteremic Streptococcus pneumoniae community-acquired pneumonia. Eur J Clin Microbiol Infect Dis 2007, 26:447-451.

8. Feldman C, Kallenbach JM, Levy H, Thorburn JR, Hurwitz MD, Koornhof $H J$ : Comparison of bacteraemic community-acquired lobar pneumonia due to Streptococcus pneumoniae and Klebsiella pneumoniae in an intensive care unit. Respiration 1991, 58:265-270.

9. Chen CW, Jong GM, Shiau JJ, Hsiue TR, Chang HY, Chuang YC, Chen CR: Adult bacteremic pneumonia: bacteriology and prognostic factors. $J$ Formos Med Assoc 1992, 91:754-759.

10. Ko WC, Paterson DL, Sagnimeni AJ, Hansen DS, Von Gottberg A, Mohapatra S, Casellas JM, Goossens H, Mulazimoglu L, Trenholme G, et al: Community-acquired Klebsiella pneumoniae bacteremia: global differences in clinical patterns. Emerg Infect Dis 2002, 8:160-166.

11. Lisboa T, Blot $S$, Waterer GW, Canalis E, de Mendoza D, Rodriguez A, Rello J: Radiologic Progression of Pulmonary Infiltrates Predicts a Worse Prognosis in Severe Community-Acquired Pneumonia Than Bacteremia. Chest 2009, 135:165-172.

12. Fine MJ, Auble TE, Yealy DM, Hanusa BH, Weissfeld LA, Singer DE, Coley CM, Marrie TJ, Kapoor WN: A prediction rule to identify low-risk patients with community-acquired pneumonia. N Engl J Med 1997, 336:243-250

13. Clinical and Laboratory Standards Institute (CLSI): Performance standards for antimicrobial susceptibility testing. 17th informational supplement. CLSI document M100-S17. 2007.

14. Palfreyman JM: Klebsiella serotyping by counter-current immunoelectrophoresis. J Hyg (Lond) 1978, 81:219-225.

15. Turton JF, Baklan H, Siu LK, Kaufmann ME, Pitt TL: Evaluation of a multiplex PCR for detection of serotypes K1, K2 and K5 in Klebsiella sp. and comparison of isolates within these serotypes. FEMS Microbiol Lett 2008, 284:247-252.

16. Yu WL, Ko WC, Cheng KC, Lee CC, Lai CC, Chuang YC: Comparison of prevalence of virulence factors for Klebsiella pneumoniae liver abscesses between isolates with capsular $\mathrm{K} 1 / \mathrm{K} 2$ and non-K1/K2 serotypes. Diagn Microbiol Infect Dis 2008, 62:1-6.

17. Yu VL, Hansen DS, Ko WC, Sagnimeni A, Klugman KP, von Gottberg A, Goossens H, Wagener MM, Benedi VJ: Virulence characteristics of Klebsiella and clinical manifestations of K. pneumoniae bloodstream infections. Emerg Infect Dis 2007, 13:986-993.

18. Tsay RW, Siu LK, Fung CP, Chang FY: Characteristics of Bacteremia Between Community-Acquired and Nosocomial Klebsiella pneumoniae Infection: Risk Factor for Mortality and the Impact of Capsular Serotypes as a Herald for Community-Acquired Infection. Arch Intern Med 2002, 162:1021-1027.

19. Chen SY, Tsai CL, Lin CH, Lee CC, Chiang WC, Wang JL, Ma MH-M, Chen SC, Chen WJ, Chang SC: Impact of liver cirrhosis on mortality in patients with community-acquired bacteremia. Diagnostic Microbiology and Infectious Disease 2009, 64:124-130.

20. Garcia-Vidal C, Fernandez-Sabe N, Carratala J, Diaz V, Verdaguer R, Dorca J, Manresa F, Gudiol F: Early mortality in patients with community-acquired pneumonia: causes and risk factors. Eur Respir J 2008, 32:733-739.

21. Jong GM, Hsiue TR, Chen CR, Chang HY, Chen CW: Rapidly fatal outcome of bacteremic Klebsiella pneumoniae pneumonia in alcoholics. Chest 1995, 107:214-217.

22. Yeh KM, Kurup A, Siu LK, Koh YL, Fung CP, Lin JC, Chen TL, Chang FY, Koh TH: Capsular serotype K1 or K2, rather than magA and rmpA, is a major virulence determinant for Klebsiella pneumoniae liver abscess in Singapore and Taiwan. J Clin Microbiol 2007, 45:466-471.

23. Fung CP, Chang FY, Lee SC, Hu BS, Kuo BI, Liu CY, Ho M, Siu LK: A global emerging disease of Klebsiella pneumoniae liver abscess: is serotype K1 an important factor for complicated endophthalmitis? Gut 2002, 50:420-424.

24. Fung CP, Siu LK: Virulence of Klebsiella pneumoniae serotype K2 should not be underestimated in K. pneumoniae liver abscess. Clin Infect Dis 2007, 45:1530-1531, author reply 1532-1533.

25. Lin YT, Chen TL, Siu LK, Hsu SF, Fung CP: Clinical and microbiological characteristics of community-acquired thoracic empyema or complicated parapneumonic effusion caused by Klebsiella pneumoniae in Taiwan. Eur J Clin Microbiol Infect Dis 2010, 29:1003-1010.

\section{Pre-publication history}

The pre-publication history for this paper can be accessed here: http://www.biomedcentral.com/1471-2334/10/307/prepub

doi:10.1186/1471-2334-10-307

Cite this article as: Lin et al:: Bacteremic community-acquired pneumonia due to Klebsiella pneumoniae: Clinical and microbiological characteristics in Taiwan, 2001-2008. BMC Infectious Diseases 2010 10:307.

\section{Submit your next manuscript to BioMed Central and take full advantage of:}

- Convenient online submission

- Thorough peer review

- No space constraints or color figure charges

- Immediate publication on acceptance

- Inclusion in PubMed, CAS, Scopus and Google Scholar

- Research which is freely available for redistribution
C Biomed Central 